\section{SEROTYPE DISTRIBUTION OF INVASIVE PNEUMOCOCCAL DISEASE IN INFANTS IN THE COMUNIDAD VALENCIANA (5 MILLION INHABITANTS), SPAIN, 2007-2010}

M. van der Heijden ${ }^{1}$, A. Rosingh ${ }^{2,3}$, E. Canton ${ }^{3}$, F. Gonzalez ${ }^{4}$, M. Gobernado ${ }^{2}$, RedMiVa (Microbiolgy surveillance network Valencia)

${ }^{1}$ University Medical Centre Groningen, Groningen, The Netherlands, '2University Hospital La Fe, ${ }^{3}$ Research centre La Fe, ${ }^{4}$ Department of Public Health, Valencia, Spain

Background: Invasive pneumococcal disease (IPD) is a cause of high mortality and morbidity worldwide. The currently available vaccines for infants include 7, (PCV7) and 13 (PCV13) serotypes.

\section{Objectives:}

1.To describe the serotype distribution of isolates of $S$. pneumoniae from IPD in infants ( $\leq 6$ years of age) in the Comunidad Valenciana from January 2007 untill 16 ${ }^{\text {th }}$ of April 2010.

2. To determine the serotype coverage of the different pneumococcal vaccines.

Materials and methods: All strains of $S$. pneumoniae causing IPD in infants in the Comunidad Valenciana, were collected from the 22 participating hospitals. Serotyping was performed by serum slide agglutination (Denka-Seiken, Tokyo, Japan).

Results: 116 Strains were collected. The most prevalent serotypes in order of prevalence were: 1 $(19,8 \%), 19 \mathrm{~A}(15,5 \%), 7 \mathrm{~F}(12,1 \%)$, and $14(8,6 \%)$. The serotype coverage of the pneumococcal vaccine in children $\leq 6$ years is $21,6 \%$ by the PCV7 and $75,0 \%$ by the PCV 13 .

\section{Conclusions:}

-The four most prevalent serotypes in infants, causing IPD in the Comunidad Valenciana during the study period were 1, 19A, 7F and 14 .

-Taking into account the high serotype coverage $(75,0 \%$ by PCV13) in infants of the most recent vaccine, it might be recommended to include the PCV13 vaccine in childhood immunization programs.

- To draw definite conclusions about including these vaccines in childhood immunization calendars, the results of safety and cost-effectiveness studies about the future vaccines should be taken into account.

PS: Data used from the Surveillance Network of the Valencia Community (MIVA network). Public Health Department, Valencia, Spain

\section{5}

\section{IMMUNOGENICITY PROFILE OF MENVEO ${ }^{\circledR}$ IN ADOLESCENTS AND ADULTS ENROLLED IN PHASE III CLINICAL TRIALS}

\author{
P. Dull ${ }^{1}$, C.J. Gill ${ }^{1}$, A. Karsten ${ }^{2}$, C. Webster ${ }^{3}$, \\ L. DeTora ${ }^{4}, A$. Anemona $^{5}$, L. Bedell ${ }^{5}$
}

${ }^{1}$ Clinical Research, ${ }^{2}$ Serology, ${ }^{3}$ Regulatory Affairs, ${ }^{4}$ Scientific Affairs, ${ }^{5}$ Biostatistics, Novartis Vaccines and Diagnostics, Cambridge, MA, USA

Background: Menveo, ${ }^{\circledR}$ a new quadrivalent meningococcal conjugate vaccine against serogroups $A, C, W-135$ and $Y$ was approved in the US and EU for persons 11- to 55-year-olds. Integrated immunogenicity data has not been presented.

Methods: Results of 4 phase 3 studies with shared inclusion/exclusion criteria and immunogenicity endpoints were evaluated. Studies compared Menveo ${ }^{\circledR}$ with previously licensed vaccines (Menactra, ${ }^{\circledR}$ Menomune ${ }^{\circledR}$ ) and evaluated sequential or co-administration of licensed adolescent vaccines (Tdap and HPV). Primary immunogenicity evaluations made 28 or 30 days postvaccination used serum bactericidal assays using human complement (hSBA); statistical noninferiority criteria were predefined.

Results: Overall, 6752 adolescents and adults were randomized to receive study vaccines at clinical centers in the United States, Italy, and Costa Rica. Comparisons to previously licensed vaccines revealed at least noninferior immunogenicity, as assessed by seroresponse for all serogroups versus both comparators as well as statistically higher results for each serogroup in various studies and age groups. Results for percentages of participants with hSBA $^{3}$ 1:8 and hSBA GMTs were supportive of seroresponse results, indicating noninferiority compared with previously licensed products. Results also supported the use of Menveo ${ }^{\circledR}$ concomitantly or sequentially with Tdap and HPV; hSBA findings were inconsistent with interference among the antigens included in co-administered or sequentially administered vaccines. 
Poster Presentation Abstracts

Conclusion: In a database of several thousand adolescents and adults, Menveo ${ }^{\circledR}$ induced significant immune responses, as assessed by hSBA, without evidence of clinically significant interference when co-administered with recommended adolescent vaccines. Responses to Menveo ${ }^{\circledR}$ were statistically non-inferior or higher compared with previously licensed comparators.

\section{6}

\section{CAT: IN HIGH-RISK INFANTS HOSPITALISED WITH BRONCHIOLITIS, IS THE USE OF PALIVIZUMAB (IG) VACCINE WARRANTED?}

S. Masand ${ }^{1,2}$, P.Y. Xiu ${ }^{2,3}$, T.B. Stoker ${ }^{3}$, M. Masand ${ }^{4}$

${ }^{1}$ King's College London Medical School, London, ${ }^{2}$ Department of Pharmacology, University of Cambridge, ${ }^{3}$ University of Cambridge Medical School, Cambridge, ' ${ }^{4}$ Department of Paediatrics, NHS Grampian Dr Gray's Hospital, Elgin, UK

Introduction: RSV bronchiolitis results in the hospitalisation of thousands of infants each year. Treatment is predominantly supportive, but palivizumab (humanised monoclonal anti-RSV antibody) administration can provide passive protection.

Objectives: To analyse experimental literature to determine whether palivizumab administration is warranted and safe in high-risk infants hospitalised with bronchiolitis. The outcomes assessed included viral titres, duration of hospitalisation, hospitalisation rates, and prospective incidence of recurrent wheezing.

Methods: Cochrane library- searched with terms "Palivizumab" and "bronchiolitis";

PubMed- searched with MeSH terms "Palivizumab" and "bronchiolitis"

Results: Palivizumab prophylaxis resulted in significant reduction in hospitalisation rates $(1 \%)$ compared to in non-prophylaxed seasons (13.5\%), and the numbers needed to treat to avoid one hospitalisation was 13 (95\% Cl: 4-8).

Prospective observation studies, suggested that prophylactic administration of palivizumab effectively reduced admissions to hospital due to re-infection.

Incidence of recurrent wheezing and physiciandiagnosed recurrent wheezing was significantly lower in antibody-treated subjects $(13 \%$ and $8 \%$ respectively) compared with untreated subjects (26\%, $\mathrm{P}=0.01$ and $16 \%, \mathrm{P}=0.011$ respectively).

Injection site reactions were uncommon $(1.8 \%$ in the placebo group compared to $2.7 \%$ in the palivizumab group). Mild or moderate elevations of ALT occurred in $1.6 \%$ of placebo and $3.6 \%$ of palivizumab recipients.

Conclusion: Palivizumab use is warranted in preterm infants hospitalised with bronchiolitis. The primary demonstrated benefit is a reduction in hospital admission rates. Further work is necessary to determine the safety of palivizumab, and the efficacy in high-risk infants (e.g. those with bronchopulmonary dysplasia). The cost-effectiveness of palivizumab administration in a UK health care setting must be assessed.

\section{7}

\section{RELATIVE EFFICACY OF LIVE ATTENUATED AND INACTIVATED INFLUENZA VACCINES IN CHILDREN AS A FUNCTION OF TIME POSTVACCINATION}

\author{
R.B. Belshe ${ }^{1}$, C.S. Ambrose ${ }^{2}$, X. Wu²,
} R. Pilsudski ${ }^{3}$

${ }^{1}$ St. Louis University School of Medicine, St. Louis, MO, ${ }^{2}$ Medlmmune, LLC, Gaithersburg, MD, USA, ${ }^{3}$ AstraZeneca, Paris, France

Aims: To examine the relative efficacy of live attenuated influenza vaccine (LAIV) and trivalent inactivated influenza vaccine (TIV) as a function of time post-vaccination from three large randomized studies that compared the efficacy of LAIV and TIV in children. LAIV is approved for use in eligible individuals 2 to 49 years of age in the U.S., Israel, Hong Kong, Macau, and South Korea.

Methods: Relative efficacy of LAIV vs. TIV against culture-confirmed influenza was calculated by timeinterval (0-4 and >4-8 months postvaccination) for matched strains, the studies' primary endpoint. Match for B viruses in Study 1 was based on genetic sequence as serology was less reliable.

Results: In each study, LAIV recipients had less influenza than TIV recipients in both time intervals and the relative efficacy of LAIV vs. TIV increased from 0-4 months to 4-8 months. 